\title{
A new set-up for the calibration of wood moisture meters
}

\author{
Jakob Fester*, Henrik Kjeldsen, Peter F. Østergaard, and Jan Nielsen
}

Danish Technological Institute, Energy and Climate, Kongsvang Allé 29, DK-8000 Aarhus C, Denmark

\begin{abstract}
A set-up has been established to calibrate wood-moisture meters at Danish Technological Institute. The set-up is based on humidification of wood in an atmosphere with constant humidity, generated by saturated salt solutions. The measurements of the reference value are made by means of the loss-on-drying method and made traceable to a primary standard for moisture mass fraction established under the EMRP project METefnet in 2015. The facility was accredited by the Danish Accreditation Fund, DANAK, in 2018. In this paper, we report on the design of the calibration set-up, the uncertainty analysis and the validation by comparison to the primary facility.
\end{abstract}

\section{Introduction}

Determination of the water content in a solid is a challenging measurement. There are various so-called reference methods available, but sometimes not only the water in the material is determined but also other liquids or volatile components, leading to errors when interpreting the measurement results. One example with this build-in-problem is the loss-on-drying method, where a sample is weighed, dried and weighted again. The water mass fraction $(W)$ is then calculated using:

$$
W=\frac{m_{1}-m_{2}}{m_{2}} \times 100 \%
$$

where $m_{1}$ and $m_{2}$ are the masses of the sample before and after drying. Depending on the type of sample, drying temperature and time etc., one may not detect the full amount of water or, alternatively, mis-assign weight losses from other volatile compounds to water when applying this method. The calibration of an instrument, traceable to SI, requires that an unambiguous measurand can be defined and an uncertainty assigned to the measurement. To be able to calibrate moisture meters, for example for wood, a link to a primary reference realizing water mass fraction therefore needs to be made to ensure true SI traceability.

Danish Technological Institute (DTI) began its activities in the field of humidity and moisture metrology in 1996 due to an emergent need from Danish Industry for accredited calibrations. A new set-up has recently been established for SI-traceable calibration of woodmoisture meters. This set-up is based on humidification of wood in an atmosphere with constant humidity, generated by saturated salt solutions. The measurements of the reference values are made by means of the loss-on-drying method and measurements are made

${ }^{*}$ Corresponding author: jafe@teknologisk.dk 
traceable to a primary standard for moisture mass fraction developed by DTI in the EMRP METefnet-project [1]. The calibration and measurement capabilities (CMC) were accredited by the national accreditation body in Denmark, DANAK, in 2018.

In this paper we report on:

- The calibration method and set-up developed for the calibration of wood moisture meters

- Validation of the method to ensure SI-traceability

- The uncertainty analysis.

\section{Calibration method}

The Unit Under Calibration (UUC) is calibrated with several wood blocks which have been conditioned by keeping them for a substantial period of time in chambers with a controlled humidity. After measuring with the UUC, the reference water content is determined by means of the loss-on-drying method and compared to the UUC reading.

\subsection{Preparation of the wood samples}

Wood of a specific type is acquired, according to the applications and wishes of the user of the wood moisture meter(s) that should be calibrated. Only a high quality of wood (e.g. without knots and mis-colored regions on the surface) is used for the calibrations, and the wood should not be impregnated which could affect the electrical resistance measured by electrical conductance moisture meters. The wood is cut into $4 \times 4 \times 10 \mathrm{~cm}$ blocks and the surfaces are polished and cleaned with dry air.

Table 1: The salts used in the DTI set-up, the nominal values of humidity (RH) they generate around room temperature and the equilibrium moisture content $(\mathrm{W}$, mass fraction of water) for spruce.

\begin{tabular}{|l|l|l|l|l|}
\hline Salt: & $\mathrm{MgCl}_{2}$ & $\mathrm{NaCl}$ & $\mathrm{KCl}$ & $\mathrm{K}_{2} \mathrm{SO}_{4}$ \\
\hline$R H, \% \mathrm{rh}$ & 33 & 76 & 86 & 98 \\
\hline$W, \%$ & 8 & 17 & 20 & 27 \\
\hline
\end{tabular}

The wood blocks are then placed in chambers with forced air circulation and a constant humidity for at least two months before they are used for calibrations. The humidity in the chambers are controlled with saturated salt solutions [1] and continuously monitored by means of calibrated capacitive humidity sensors. The relative humidity is held constant with a requirement of maximum fluctuations of $\pm 1.5 \% \mathrm{rh}$ during the preparation period. This criterium imposes a corresponding requirement for temperature stability due to the temperature dependence of equilibrium relative humidity $(R H)$ using salt solutions [2]. In practice $\pm 2{ }^{\circ} \mathrm{C}$ is sufficient for this purpose (see [2]). The relation between the equilibrium relative humidity and the equilibrium water content of the sample is given by the moisture sorption isotherm but is difficult to predict for several reasons. Firstly, the sorption isotherm for wood appears to have a significant adsorption/desorption hysteresis. This means that the equilibrium moisture content depends on whether the wood is drying or being wetted, when placed in the chamber. Secondly the sorption isotherm is dependent on the variety of wood. The latter can of course be solved by determining the sorption isotherm experimentally for 
each type of sample, but this is a very costly and time-consuming process. The values stated in Table 1 are therefore estimates based on experience.

\subsection{Measurement strategy}

Initially the mass, $m_{1}$, before, of the selected wood blocks is measured with a calibrated balance. The time of measurement is registered. Measurements are now performed with the UUC in four sections, on each side of the wood block (Figure 1). In each section, measurements are performed both parallel and perpendicular to the wood grain. This adds up to 32 measurements over the 4 sides of the block. The average of these measurements is reported in the certificate. In case the UUC is of the pin-type, the measurement depth is agreed with the customer. Finally, measurement of the mass of the block is repeated $\left(m_{1}\right.$, after $)$ in order to estimate the water loss or gain due to handling. An average mass, $m_{1}$, is calculated as the average of $m_{1}$, before and $m_{1}$, after and used for determining the moisture content reference value $(W)$.

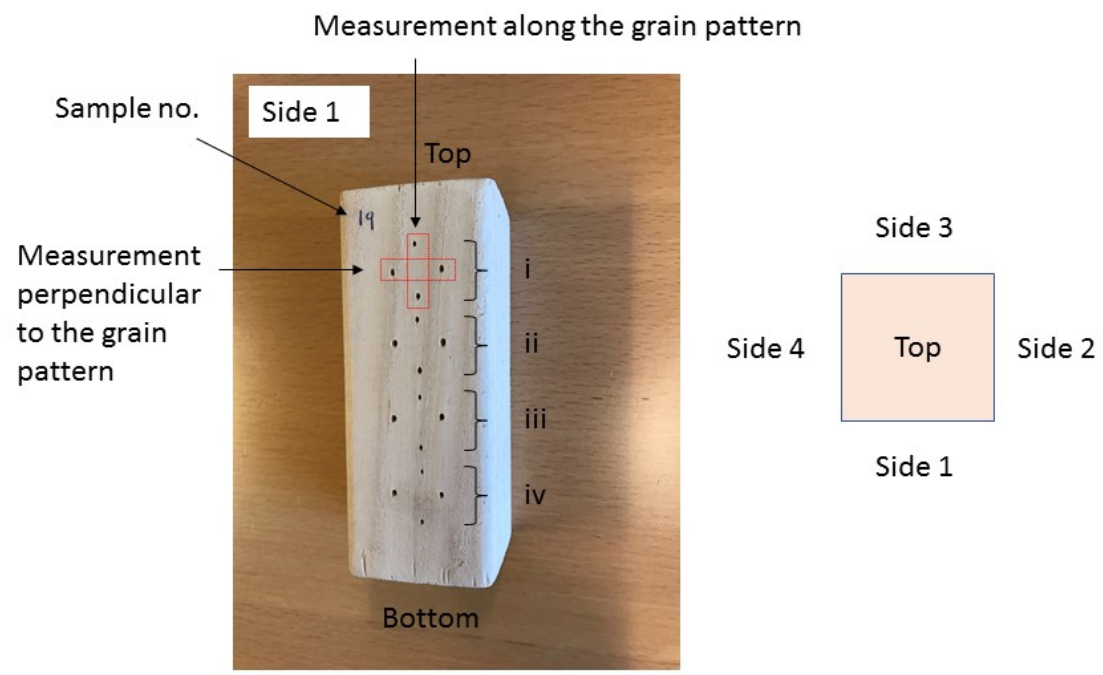

Figure 1: Wood block, spruce, after being used to calibrate a wood moisture meter of the pin-type.

\subsection{Determination of the water content}

The water mass fraction is determined by the loss-on-drying method, roughly as laid out in the documentary standard ISO 4470 [3]. The wood blocks used for the calibration are dried in an oven at $\left(103{ }^{\circ} \mathrm{C} \pm 2{ }^{\circ} \mathrm{C}\right)$ in 20 hours. Immediately after removing the blocks from the oven, they are placed in a drying chamber (desiccator) while cooling down to room temperature. The mass of the block is measured $\left(m_{2}\right)$ and the water mass fraction calculated according to (1). 


\section{Validation of the method}

\subsection{A primary set-up for determining moisture mass fraction}

For validating the method, a comparison was made between the loss-on-drying method and an in-house, primary measurement standard for moisture mass fraction that has been in operation since 2015 .

In the primary standard, the total mass of evaporated water from a sample placed in a heated measurement chamber is determined by integrating the amount of water vapour in exhaust air over the drying period. The mass of water vapour released is calculated from the measured dew point temperature, air temperature and air flow rate. The dew point temperature is determined using a combination of a capacitive sensor and a chilled mirror hygrometer $(\mathrm{CMH})$, which enables measurements in the presence of possible contaminants evaporated from the sample. The traceability to SI is obtained through traceable measurements of air-flow, air temperature and dew point temperature. The relative expanded uncertainty of $1.6 \%$ has been demonstrated in intercomparisons $[4,5]$.

\subsection{Results}

\subsubsection{Adsorption and desorption curves}

The set-up was validated using samples made from spruce. The samples were cut and prepared according to the procedure specified in 2.1. Four samples were placed in every chamber and their individual weights monitored over time. Each piece of wood was weighed two times every week over a period of 2 months. The relative weights compared to the initial weight (defined as 1) was then calculated and an average for every preparation environment is shown as a function of time in Figure 2.

From the weight curves (Figure 2), it was estimated that the adsorption and desorption processes had finished after 30 days. However, in the case of $98 \%$ relative humidity, the results indicate that 60 days / two months are needed to make sure that all the processes of water uptake have terminated and levelled out to a flat weight curve.

We tested for the case of inhomogeneous distribution of moisture inside the wood samples which might potentially occur as a consequence of insufficient adsorption (or desorption) time in the preparation chambers. To carry out this test, we assessed if the water content was dependent on the depth from the wood surface by cutting several samples along the wood fibers in three parallel and flat pieces. No systematic moisture content profile was observed. Hence, the test confirms that the indicated preparation time of two months in the humidity chambers is sufficient to reach a full humidification through the wood samples.

\subsubsection{Influence of laboratory air during calibration}

A variety of tests were performed to analyze several potential issues in the experimental calibration procedure.

We evaluated the degree of water exchange with the atmosphere during measurements with the UUC. The weights of individual wood samples were monitored while they rested in laboratory air (calibration conditions). The resulting development of their masses is shown in Figure 3. It can be concluded that after $30 \mathrm{~min}$, representative of a realistic series of measurements with the UUC, a significant exchange of moisture with the surrounding air has 
taken place. For pieces of wood with the highest moisture content (nominal $27 \%(W)$ ), the experiment shows that, after $30 \mathrm{~min}$, the samples have lost $0,8 \%$ of their mass relative to the dry weight. In comparison, the change in water content for the samples prepared at $33 \% \mathrm{rh}$, $76 \%$ rh and $86 \%$ rh, amounts to $+0.1 \%,-0.3 \%$ and $-0.4 \%$.

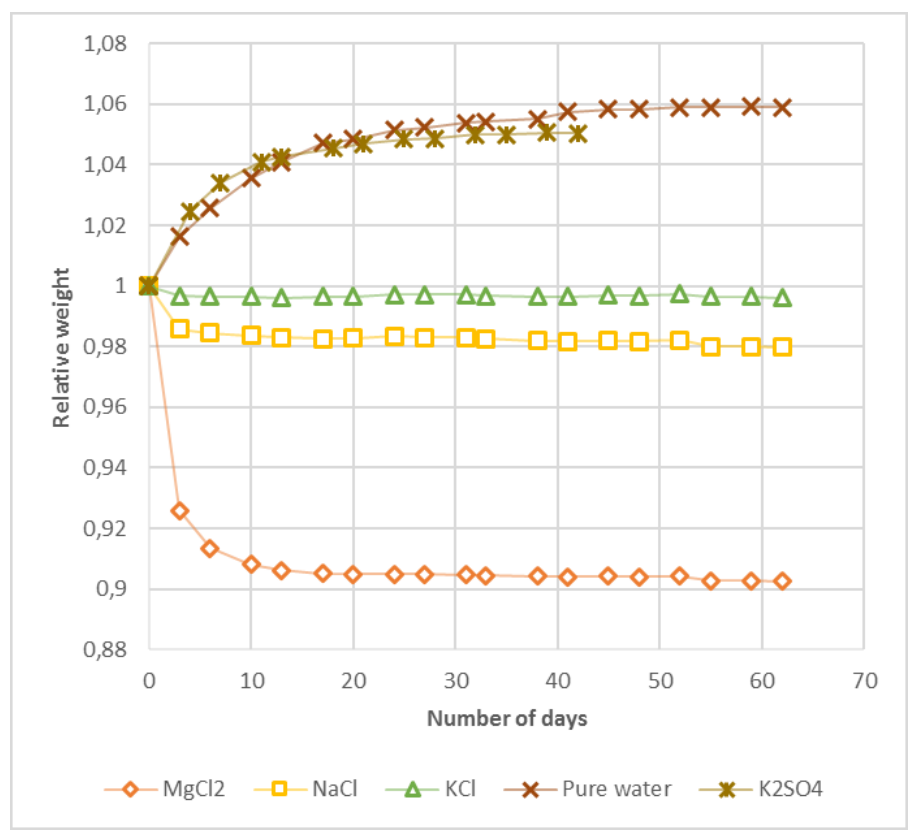

Figure 2: Weight curves for wood samples during humidification by storage in atmospheric environments regulated by equilibrium salt solutions at room temperature $\left(\mathrm{MgCl}_{2}: 33 \% \mathrm{rh}, \mathrm{NaCl}\right.$ : $76 \% \mathrm{rh}, \mathrm{KaCl}: 86 \% \mathrm{rh}, \mathrm{K}_{2} \mathrm{SO}_{4}: 98 \% \mathrm{rh}$ and pure water : $100 \% \mathrm{rh}$ ).

The significant exchange of moisture with air during calibration has to be considered in the analysis. The point is taken into account in the procedure described in 2.2 by determining the wet mass both before and after the UUC-measurements and using an average value $\left(m_{1}\right.$ in equation (1)) to calculate the water content, $W$, by the loss-on-drying method.

We note that the water exchange with surrounding air will also affect the recorded readings of single UUC measurements during calibration, which should theoretically vary systematically in the course of the calibration of up to 30 minutes. For analysis, an average of these UUT values is used to determine the error compared to the reference value. This procedure, using the averages of both readings and wet mass, $m_{1}$, before and after measurement ensures that the instrument reading and reference value are still comparable. However, the variation of UUC measurements due to the changing mass in laboratory air contributes to the total uncertainty, where it is going to enter through the repeatability, $u$ (rep), i.e. the standard uncertainty of the UUC measurements (see 4). 


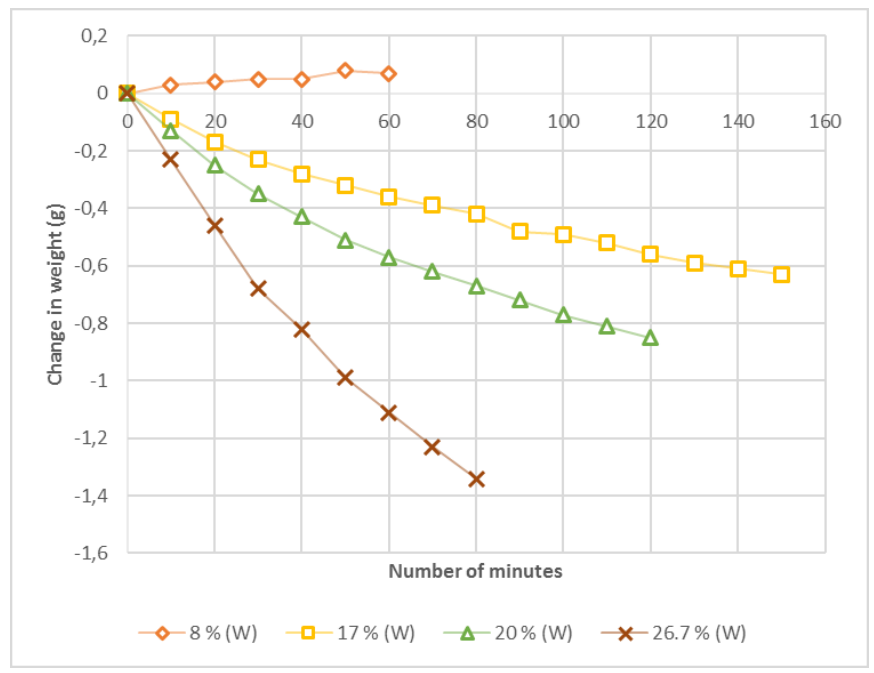

Figure 3: Adsorption- and desorption curves for samples exposed to laboratory conditions $\left(21^{\circ} \mathrm{C} / 49\right.$ $\%$ rh). The curves correspond to individual wood samples of different nominal moisture contents (W) using the four salts and humidity conditions stated in Table 1.

\subsubsection{Corrections to the dry mass}

In the determination of the dry mass, a first correction must be made, originating from a water uptake during the cooling time ( 2 hours) before the measurement on the balance is carried out after oven drying. To estimate the correction, we monitored the weight of a sample situated in the desiccator for cooling. The desiccator was loaded with a silica gel desiccant to generate an environment with minimal moisture, however not dry enough to completely prevent moisture adsorption in the dried wood. As can be seen in Figure 4, the sample increases its weight by approximately $0.2 \mathrm{~g}$ during the first 40 hours. According to this weight curve, we estimate that the sample adsorbs $0.05 \mathrm{~g}$ of water during the cooling time of 2 hours used in the experimental procedure. Hence, the dry mass is corrected by the amount of -0.05 $\mathrm{g}$ and an associated uncertainty of $0.05 \mathrm{~g} / \sqrt{3}$ is added to the uncertainty budget ( $u\left(m_{2, \text { cooling }}\right)$ ).

It has previously been emphasized that, also, the influence of transport conditions is important to consider when handling samples in moisture measurements [6]. Hence, a second influence on the dry mass is a potential water uptake during transportation from the oven to the laboratory balance (after cooling down in desiccator). Indeed, the mass difference of 6 wood samples before and after transportation inside the laboratory building (from the location of the oven to the laboratory balance) yielded weight gains from $0.04 \mathrm{~g}$ to $0.07 \mathrm{~g}$. Since the actual weight gain is in theory dependent on current atmospheric conditions (temperature and relative humidity), a standard correction cannot be made in this case, however an uncertainty on $0.1 \mathrm{~g}$ was included in the uncertainty budget $u\left(m_{2}\right.$,transport $\left.)\right)$ to fully cover a worst case scenario. 


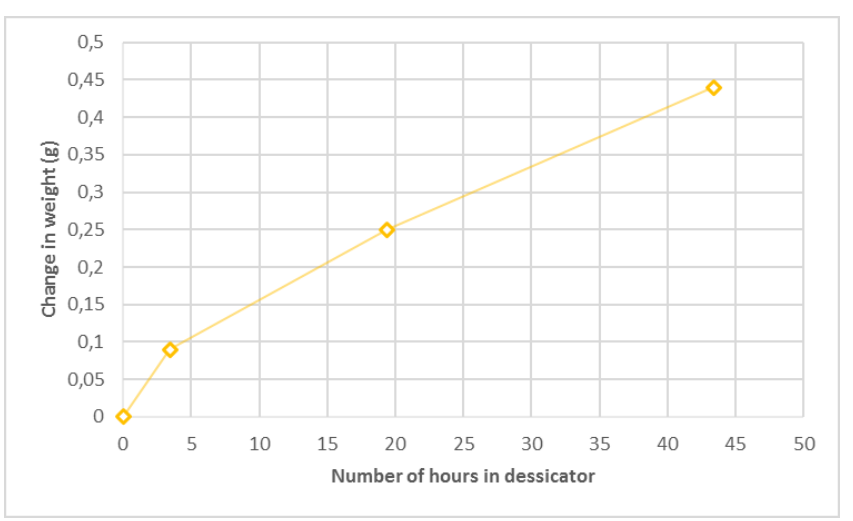

Figure 4: Change in weight of a dried wood sample (spruce) during storage in desiccator after oven treatment. The original dry weight was $77.19 \mathrm{~g}$.

\subsubsection{Examples of calibration}

As a typical example, a pin-type instrument (TimberMaster BLD5605 Plus Moisture Meter) was calibrated using a probing depth of $6 \mathrm{~mm}$ at nominal moisture content in a spruce sample of nominal $17 \%$ moisture content.

Over the 32 measuring points (see Figure 1), the readings varied between minimum and maximum values of $13.8 \%$ to $16.6 \%$ moisture content with an average of $15.2 \%$. This resulted in an error of $-2.1 \%(W)$ and an uncertainty of $2.3 \%(W)$ for a 95,45\% confidence interval. The determined moisture content was $17.3 \%$ in this case. The individual uncertainty components for this example calibration are given in Table 2

We emphasize, that in the case of a pin-type UUC, we found that the probing depth, i.e. how far the pins protrude into the wood, is a critical parameter that is strongly affecting the reading on an instrument. Systematic measurements using adjustment of the probing depth by stacking of non-conductive blocks between the instrument and wood surface, showed that the reading varied by $2 \%$ up to $4 \%$ moisture content, depending on the nominal water content $(W)$. Therefore, it is of critical importance that the probing depth is held constant throughout measurements and reported on the calibration certificate. Small variations in probing depth and the uncertainty in measurement stemming from this issue enters the uncertainty budget through the repeatability $(u($ rep $))$.

\section{Uncertainty analysis}

The uncertainty analysis was made according to the GUM method [7]. An example of an uncertainty budget is shown in Table 2 and includes the following components:

$u$ (val): The uncertainty due to the validation of the method (see 3.2). This uncertainty comprises $2.0 \%$ of the mass of water in the wood sample, and is included in the uncertainty budget as $2.0 \%$ of the reference value, $W$.

$u$ (read): The uncertainty of reading, in best case this is half the resolution divided by square root three, assuming a uniform distribution. 
$u$ (rep): The repeatability of the measurement. As laid out in section 2.2, up to 32 measurements are made during the calibration. The uncertainty is calculated as the experimental standard uncertainty.

$u\left(m_{1, \text { before }}\right), u\left(m_{1, \text { after }}\right)$ and $u\left(m_{2}\right)$ :

The uncertainty of the determination of mass, before ( $m_{1, \text { before }}$ and $\left.m_{1, \text { after }}\right)$ and after drying $\left(m_{2}\right)$, where

$$
m_{1}=\left(m_{1, \text { before }}+m_{1, \text { after }}\right) / 2,
$$

referring to "before" and "after" calibration measurements with the UUC. This uncertainty is the uncertainty of calibration of the balance, including eccentricity and linearity.

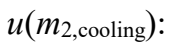

An uncertainty component related with a correction to the dry mass, $\delta_{m_{2}}$,air, given by

$$
m_{2}=m_{2, \text { meas }}+\delta_{m_{2}, a i r}
$$

where $m_{2, \text { meas }}$ is the measured value on the balance and $\delta_{m_{2}, \text { air }}$ is due to any gain in weight of the wood block while cooling down in desiccator after drying in oven. This uncertainty component has been estimated based on experiments (see 3.2.3).

$u\left(m_{2, \text { transport }):}\right.$

An uncertainty component related with a correction to the dry mass due to water uptake during transport in the time from removing it from the desiccator to determining its mass.

The combined standard uncertainty, $u_{c}(W)$, is the square root of the sum of squares of the individual standard uncertainties and associated with an effective number of degrees of freedom that traces back to the number of measured points on the wood sample minus one [8]. The expanded uncertainty is found by multiplying $u_{c}(W)$ with the coverage factor, $k$, obtained from an inverse T-distribution function or table with $95,45 \%$ confidence interval and the calculated number of effective degrees of freedom.

The two largest and dominating uncertainty contributions are $u$ (rep), repeatability, and $u$ (val), validation. While the uncertainty stemming from the validation method cannot be further reduced (see 3.1), the repeatability is among other factors depending on exchange with moisture in the laboratory air during measurements, as well as the homogeneity of the wood in a specific wood sample and is therefore subject to variations.

Since the uncertainty component from repeatability can increase dramatically in case of inhomogeneous wood, it is necessary to impose an upper limit for the variation of the instrument readings. If the limit is crossed, the homogeneity of the respective wood sample is judged to be inappropriate for calibration and it will be discarded, and a new sample applied instead. Alternatively, in less critical cases, a few single measurement points outside the range can be omitted in the analysis if the cause can be identified, e.g. if the pins hit a knot or irregularity. This will affect the number of degrees of freedom regarding the repeatability (see Table 2), but with a limited impact on the resulting 95,45\% confidence interval for the calculated error. 
Table 2: Example of an uncertainty budget for the calibration of a pin-type moisture meter at nominal $17 \%$ water content. $\mathrm{u}_{\mathrm{c}}$ is the combined standard uncertainty.

\begin{tabular}{|l|l|l|l|l|l|}
\hline $\begin{array}{l}\text { Uncertainty } \\
\text { component }\end{array}$ & $u\left(x_{i}\right)$ & unit & $\frac{\partial W}{\partial x_{i}}$ & $u\left(W_{i}\right)$ & $\begin{array}{l}\text { deg of } \\
\text { freedom }\end{array}$ \\
\hline$u($ val $)$ & 0.30 & absolute $\%$ & 1 & 0.35 & $\infty$ \\
\hline$u($ read $)$ & 0.029 & absolute $\%$ & 1 & 0.03 & $\infty$ \\
\hline$u($ rep $)$ & 1.0 & absolute $\%$ & 1 & 1.0 & 31 \\
\hline$u\left(m_{1, \text { before })}\right.$ & 0.10 & $\mathrm{~g}$ & 0.59 & 0.06 & $\infty$ \\
\hline$u\left(m_{1, \text { after })}\right.$ & 0.10 & $\mathrm{~g}$ & 0.59 & 0.06 & $\infty$ \\
\hline$u\left(m_{2}\right)$ & 0.10 & $\mathrm{~g}$ & -1.38 & -0.14 & $\infty$ \\
\hline$u\left(m_{2, \text { cooling }}\right)$ & 0.03 & $\mathrm{~g}$ & -1.38 & -0.06 & $\infty$ \\
\hline$u\left(m_{2, \text { trainsport }}\right)$ & 0.10 & $\mathrm{~g}$ & -1.38 & -0.14 & $\infty$ \\
\hline$u_{c}(W)$ & & absolute $\%$ & & 1.08 & 31 \\
\hline$U(95,45 \%)$ & & absolute $\%$ & & 2.3 & \\
\hline
\end{tabular}

\section{Conclusions}

In conclusion, we have demonstrated a new set-up for calibration of wood moisture meters by measurement on wood samples prepared to contain different levels of moisture content, determined using a loss-on-drying method. Traceability is achieved by validation of the procedure with an in-house primary standard that directly measures the total mass of evaporated water from a sample. The set-up has in a first step been validated for measurement on spruce and pin-type moisture meters, but more types of wood and instruments are planned to be included in future work.

This study was supported by a grant from Danish Technological Institutes performance contract 20162018, entered with the Danish Agency for Institutions and Educational Grants, under The Ministry of Higher Education and Science.

\section{References}

1. P. Østergaard and J. Nielsen, Int J Thermophys 39:1 (2018)

2. OIML R 121 (1996), https://www.healthpack.net/files/SaltSolutions.pdf, accessed 8 July 2019

3. ISO 4470:1981 first edition

4. M. Heinonen, S. Bell, B. Il Choi, G. Cortellessa, V. Fernicola, E. Georgin, D. Hudoklin, G. V. Ionescu, N. Ismail, T. Keawprasert, M. Krasheninina, R. Aro, J. Nielsen, S. Oğuz Aytekin, P. Österberg, J. Skabar, and R. Strnad, Int J Thermophys 39:1 20 (2018) 
5. S. Bell et al., 17th International Congress of Metrology, p. 15003 (EDP Sciences, 2015). DOI: 10.1051/metrology/201515003 (2015)

6. Pálková, Zuzana, et al., Int J Thermophys 38:10 (2017): 153

7. JCGM 2008, https://www.bipm.org/utils/common/documents/jcgm/JCGM_100_2008_E.pdf, accessed 8 July 2019

8. EA-4/02 M: 1999, https://www.renar.ro/files/OEC/download/accreditation-guides/ea-402-m.pdf, accessed 11 July 2019 